Network Working Group

Request for Comments: 5287

Category: Standards Track
A. Vainshtein ECI Telecom $Y(J)$. Stein RAD Data Communications August 2008

\title{
Control Protocol Extensions for the Setup of Time-Division Multiplexing (TDM) Pseudowires in MPLS Networks
}

\section{Status of This Memo}

This document specifies an Internet standards track protocol for the Internet community, and requests discussion and suggestions for improvements. Please refer to the current edition of the "Internet Official Protocol Standards" (STD 1) for the standardization state and status of this protocol. Distribution of this memo is unlimited.

Abstract

This document defines extension to the Pseudowire Emulation Edge-toEdge (PWE3) control protocol RFC 4447 and PWE3 IANA allocations RFC 4446 required for the setup of Time-Division Multiplexing (TDM) pseudowires in MPLS networks.

Table of Contents

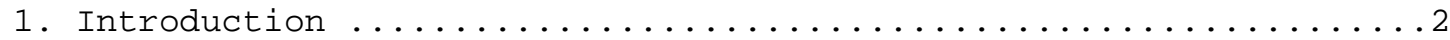

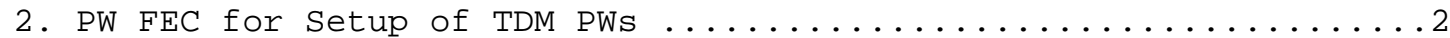

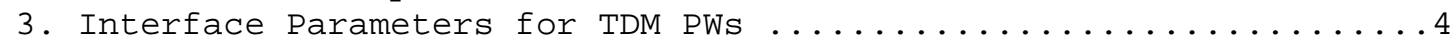

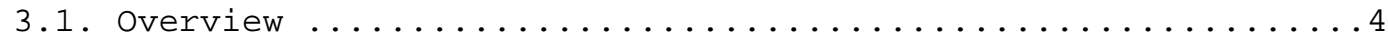

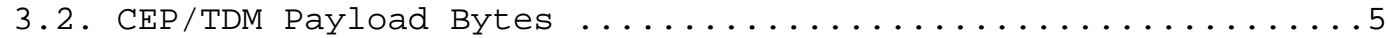

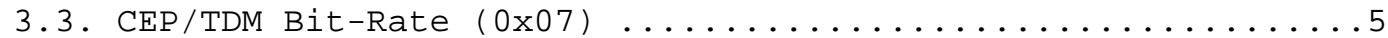

3.4. Number of TDMoIP AAL1 Cells per Packet ............

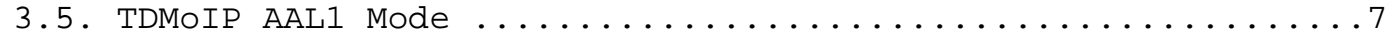

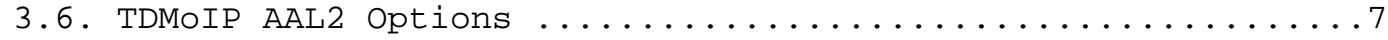

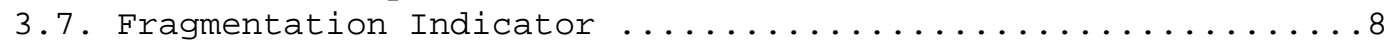

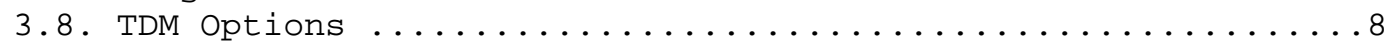

4. Extending CESOPSN Basic NxDSO Services with CE

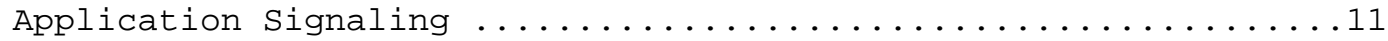

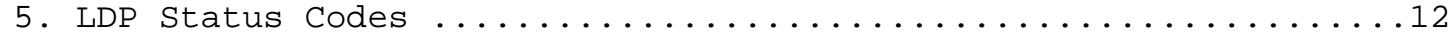

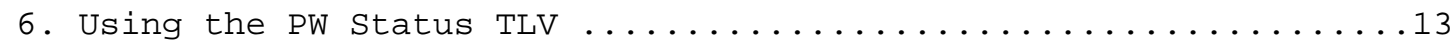

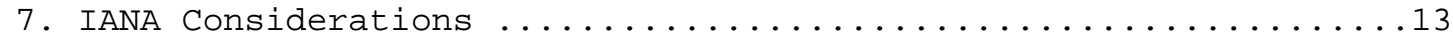

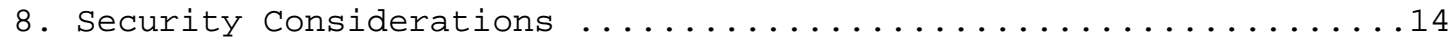

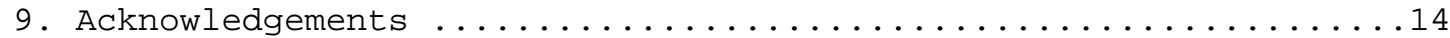

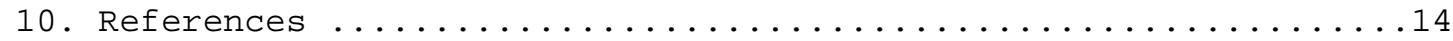

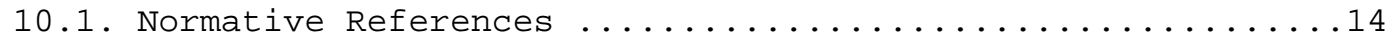

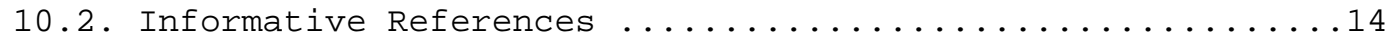




\section{Introduction}

This document defines an extension to the PWE3 control protocol [RFC4447] and PWE3 IANA allocations [RFC4446] required for the setup of TDM pseudowires in MPLS networks.

Structure-agnostic TDM pseudowires have been specified in [RFC4553], and structure-aware ones have been specified in [RFC5086] and [RFC5087].

[RFC4447] defines extensions to the Label Distribution Protocol (LDP) [RFC5036] that are required to exchange PW labels for PWs emulating various Layer 2 services (Ethernet, Frame Relay (FR), Asynchronous Transfer Mode (ATM), High-Level Data Link Control (HDLC), etC.). The setup of TDM PWs requires both interpretation of the existing information elements of these extensions and exchange of additional information.

The setup of TDM PWs using L2TPV3 will be defined in a separate document.

The status of attachment circuits of TDM PWs can be exchanged between the terminating Provider Edges (PES) using the PW Status mechanism defined in [RFC4447] without any changes. However, usage of this mechanism is NOT RECOMMENDED for TDM PWs since the indication of the status of the TDM attachment circuits is carried in-band in the data plane.

The key words "MUST", "MUST NOT", "REQUIRED", "SHALL", "SHALL NOT", "SHOULD", "SHOULD NOT", "RECOMMENDED", "MAY", and "OPTIONAL" in this document are to be interpreted as described in [RFC2119].

\section{PW FEC for setup of TDM PWs}

[RFC4447] uses the LDP Label Mapping message [RFC5036] for advertising the FEC-to-PW Label binding, and defines two types of PW Forwarding Equivalence Classes (FECs) that can be used for this purpose:

1. PWId FEC (FEC 128). This FEC contains:
a) PW type
b) Control bit (indicates presence of the control word)
c) Group ID
d) PW ID 
e) Interface parameters Sub-TLV

2. Generalized PW FEC (FEC 129). This FEC contains only:

a) PW type

b) Control bit

c) Attachment Group Identifier (AGI), Source Attachment Individual Identifier (SAII), and Target Attachment Individual Identifier (TAII) that replace the PW ID

The Group ID and the Interface Parameters are contained in separate TLVs, called the PW Grouping TLV and the Interface Parameters TLV.

Either of these types of PW FEC MAY be used for the setup of TDM PWs with the appropriate selection of PW types and interface parameters.

The PW types for TDM PWs are allocated in [RFC4446] as follows:

- 0x0011 Structure-agnostic E1 over Packet [RFC4553]

- 0x0012 Structure-agnostic T1 (DS1) over Packet [RFC4553]

- 0x0013 Structure-agnostic E3 over Packet [RFC4553]

- 0x0014 Structure-agnostic T3 (DS3) over Packet [RFC4553]

- 0x0015 CESOPSN basic mode [RFC5086]

- 0x0016 TDMOIP AAL1 mode [RFC5087]

- 0x0017 CESOPSN TDM with CAS [RFC5086]

- 0x0018 TDMOIP AAL2 mode [RFC5087]

The two endpoints MUST agree on the PW type, as both directions of the $\mathrm{PW}$ are required to be of the same type.

The Control bit MUST always be set for TDM PWs since all TDM PW encapsulations always use a control word.

PW type 0x0012 MUST also be used for the setup of structure-agnostic TDM PWs between a pair of J1 attachment circuits (see [RFC4805]). 


\section{Interface Parameters for TDM PWs}

\subsection{Overview}

The interface parameters that are relevant for the setup of the TDM PWs are listed below.

\begin{tabular}{|c|c|c|c|}
\hline Interface Parameter & Sub-TLV ID & Length & Description \\
\hline ---------------------- & ------------- & & -------------1 \\
\hline CEP/TDM Payload Bytes & $0 \times 04$ & 4 & Section 3.2 \\
\hline----------------------- & ------ & ----- & ------------ \\
\hline CEP/TDM Bit-Rate & $0 \times 07$ & 6 & Section 3.3 \\
\hline----------------------- & ----- & & -------ー--- \\
\hline $\begin{array}{l}\text { Number of TDMoIP AAL1 } \\
\text { Cells per Packet }\end{array}$ & $0 \times 0 E$ & 4 & Section 3.4 \\
\hline------------------------- & $-------=----$ & --- & ----------- \\
\hline TDMoIP AAL1 Mode & $0 \times 10$ & 4 & Section 3.5 \\
\hline 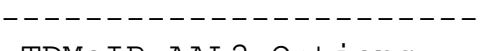 & -----1 & -------- & -----------1 \\
\hline TDMoIP AAL2 Options & $0 \times 11$ & $\begin{array}{c}8 \text { or } \\
\text { larger } \\
\text { see note }\end{array}$ & Section 3.6 \\
\hline $\begin{array}{l}\text { Fragmentation } \\
\text { Indicator }\end{array}$ & $0 \times 09$ & $\begin{array}{l}-------- \\
4\end{array}$ & Section 3.7 \\
\hline TDM Options & $\begin{array}{l}0 \times 0 \mathrm{~B} \\
\mathrm{O}\end{array}$ & $\begin{array}{c}-1,8, \\
\text { or } 12\end{array}$ & Section 3.8 \\
\hline
\end{tabular}

If not explicitly indicated otherwise in the appropriate description, the value of the interface parameter is interpreted as an unsigned integer of the appropriate size (16 or 32 bits).

Note: The length of basic TDMoIP AAL2 Options interface parameter is 8 bytes, and when the optional Channel ID (CID) mapping bases field is used, there is one additional byte for each trunk transported. Thus, if 1 trunk is being supported, this message occupies 9 bytes. Since there can be no more than 248 CIDs in a given PW, this can never exceed 256 (this when each channel comes from a different trunk). 248 channels translates to less than 9 Els, and so, for this case, the length is 
no more than 17 bytes. A single PE is not required to support more than 10 AAL2 PWs (i.e., up to 2480 individual channels, which is more than carried by a fully populated STM1). Thus, the memory required to store all the AAL2 mapping information is typically between 80 and 170 bytes per PE.

\subsection{CEP/TDM Payload Bytes}

This parameter is used for the setup of all SAToP and CESOPSN PWs (i.e., PW types 0x0011, 0x0012, 0x0013, 0x0014, 0x0015, and 0x0017) and employs the following semantics:

1. The two endpoints of a TDM PW MUST agree on the same value of this parameter for the PW to be set up successfully.

2. Presence of this parameter in the PWId FEC or in the Interface Parameters Field TLV is OPTIONAL. If this parameter is omitted, default payload size defined for the corresponding service (see [RFC4553], [RFC5086]) MUST be assumed.

3. For structure-agnostic emulation, any value consistent with the MTU of the underlying PSN MAY be specified.

4. For CESOPSN PWS:

a) The specified value $P$ MUST be an integer multiple of $N$, where $N$ is the number of timeslots in the attachment circuit.

b) For trunk-specific NxDSO with CAS:

i) (P/N) MUST be an integer factor of the number of frames per corresponding trunk multiframe (i.e., 16 for an E1 trunk and 24 for a T1 or J1 trunk).

ii) The size of the signaling sub-structure is not accounted for in the specified value $P$.

5. This parameter MUST NOT be used for the setup of TDMoIP PWs (i.e., PWs with PW types 0x0016 and 0x0018).

\subsection{CEP/TDM Bit-Rate $(0 \times 07)$}

This interface parameter represents the bit-rate of the TDM service in multiples of the "basic" $64 \mathrm{kbit} / \mathrm{s}$ rate. Its usage for all types of TDM PWs assumes the following semantics: 
1. This interface parameter MAY be omitted if the attachment circuit bit-rate can be unambiguously derived from the PW type (i.e., for structure-agnostic emulation of E1, E3, and T3 circuits). If this value is omitted for the structure-agnostic emulation of $\mathrm{T} 1 \mathrm{PW}$ type, the basic emulation mode MUST be assumed.

2. If present, only the following values MUST be specified for structure-agnostic emulation (see [RFC4553]:

a) Structure-agnostic E1 emulation - 32

b) Structure-agnostic T1 emulation:

i) MUST be set to 24 in the basic emulation mode

ii) MUST be set to 25 for the "Octet-aligned T1" emulation mode

c) Structure-agnostic E3 emulation - 535

d) Structure-agnostic T3 emulation - 699

3. For all kinds of structure-aware emulation, this parameter MUST be set to $N$, where $\mathrm{N}$ is the number of DSO channels in the corresponding attachment circuit.

Note: The value 24 does not represent the actual bit-rate of the T1 or J1 circuit $(1,544 \mathrm{Mbit} / \mathrm{s})$ in units of $64 \mathrm{kbit} / \mathrm{s}$. The values mentioned above are used for convenience.

Note: A 4-byte space is reserved for this parameter for compatibility with [RFC4842].

\subsection{Number of TDMoIP AAL1 Cells per Packet}

This parameter MAY be present for TDMoIP AAL1 mode PWs (PW type 0x0016) and specifies the number of 48-byte AAL1 PDUs per MPLS packet. Any values consistent with the MTU of the underlying PSN MAY be specified. If this parameter is not specified, it defaults to 1 PDU per packet for low bit-rates (CEP/TDM Bit-Rate less than or equal to 32), and to 5 for high bit-rates (CEP/TDM Bit-Rate of 535 or 699). 


\subsection{TDMOIP AAL1 Mode}

This parameter MAY be present for TDMoIP AAL1 mode PWs (PW type 0x0016) and specifies the AAL1 mode. If this parameter is not present, the AAL1 mode defaults to "structured". When specified, the values have the following significance:

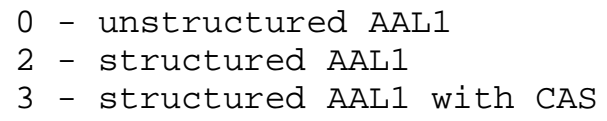

The two endpoints MUST agree on the TDMoIP AAL1 mode.

\subsection{TDMOIP AAL2 Options}

This parameter MUST be present for TDMoIP AAL2 mode PWs (PW type 0x0018) and has the following format:

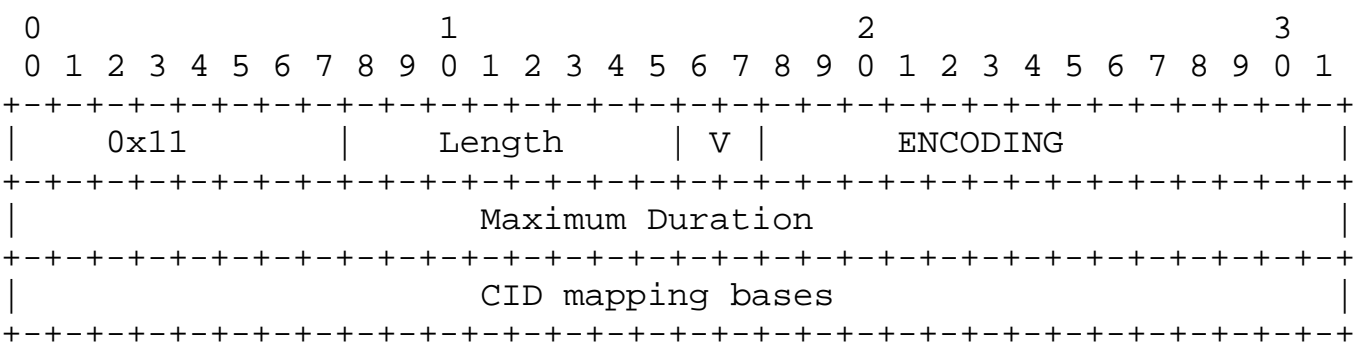

The fields in this parameter are defined as follows:

$\mathrm{V}$ defines the Voice Activity Detection (VAD) capabilities. Its values have the following significance:

0 means that activity is only indicated by signaling.

1 means that voice activity detection is employed.

3 means this channel is always active. In particular, this channel may be used for timing recovery.

Encoding specifies native signal processing performed on the payload. When no native signal processing is performed (i.e., G.711 encoding), this field MUST be zero. Other specific values that can be used in this field are beyond the scope of this specification, but the two directions MUST match for the PW setup to succeed. 
Maximum Duration specifies the maximum time allowed for filling an AAL2 PDU, in units of 125 microseconds. For unencoded 64 kbps channels, this numerically equals the maximum number of bytes per PDU and MUST be less than 64. For other encoding parameters, larger values may be attained.

CID mapping bases is an OPTIONAL parameter; its existence and length are determined by the length field. If the mapping of AAL2 CID values to a physical interface and time slot is statically configured, or if AAL2 switching [Q.2630.1] is employed, this parameter MUST NOT appear. When it is present, and the channels belong to $\mathrm{N}$ physical interfaces (i.e., $\mathrm{N}$ Els or T1s), it MUST be $\mathrm{N}$ bytes in length. Each byte represents a number to be subtracted from the CID to get the timeslot number for each physical interface. For example, if the CID mapping bases parameter consists of the bytes 20 and 60, this signifies that timeslot 1 of trunk 1 corresponds to CID 21, and timeslot 1 of trunk 2 is called 61 .

\subsection{Fragmentation Indicator}

This interface parameter is specified in [RFC4446], and its usage is explained in [RFC4623]. It MUST be omitted in the FEC of all TDM PWs excluding trunk-specific NxDSO services with CAS using the CESoPSN encapsulation. In the case of these services, it MUST be present in the PW FEC if the payload size specified value $P$ differs from $\mathrm{Nx}$ (number of frames per trunk multiframe).

\subsection{TDM Options}

This is a new interface parameter. Its Interface Parameter ID (Ox0B) has been assigned by IANA, and its format is shown in Figure 1 below:

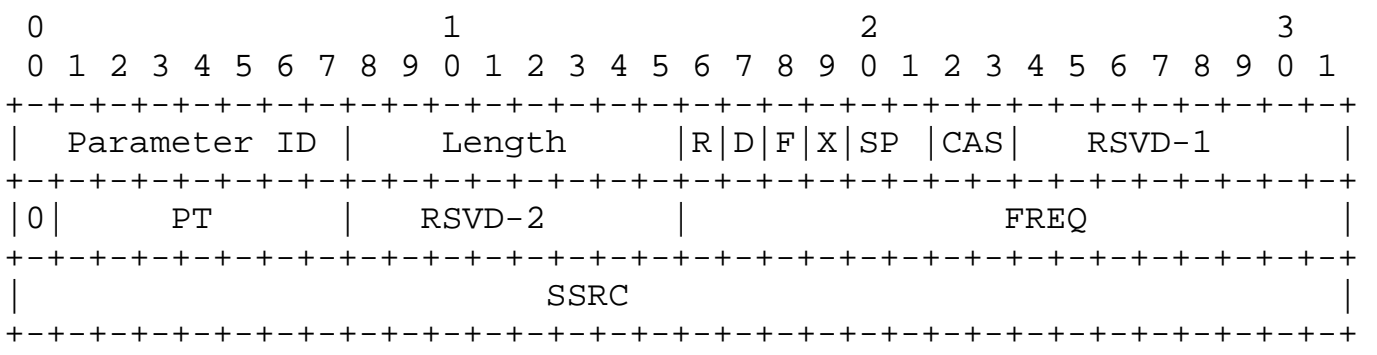

Figure 1. Format of the TDM Options Interface Parameter Sub-TLV

The fields shown in this diagram are used as follows:

Parameter ID Identifies the TDM PW Options interface parameter, $0 \times 0 B$. 
Length

$\mathrm{R}$

D

$F, \quad X$

SP

Vainshtein \& Stein
4, 8, or 12 (see below).

The RTP Header Usage bit: if set, indicates that the PW endpoint distributing this FEC expects to receive RTP header in the encapsulation. RTP header will be used only if both endpoints expect to receive it. If this bit is cleared, Length MUST be set to 4; otherwise, it MUST be either 8 or 12 (see below). If the peer $\mathrm{PW}$ endpoint cannot meet this requirement, the Label Mapping message containing the FEC in question MUST be rejected with the appropriate status code (see Section 4 below).

The Differential timestamping Mode bit: if set, indicates that the PW endpoint distributing this FEC expects the peer to use Differential timestamping mode in the packets sent to it. If the peer PW endpoint cannot meet this requirement, the Label Mapping message containing the FEC in question MUST be rejected with the appropriate status code (see section 4 below).

Reserved for future extensions. MUST be cleared when distributed and MUST be ignored upon reception.

Encodes support for the CESoPSN signaling packets (see [RFC5086]):

o '00' for PWs that do not use signaling packets

- '01' for CESOPSN PWs carrying TDM data packets and expecting Customer Edge (CE) application signaling packets in a separate $\mathrm{PW}$

- '10' for a PW carrying CE application signaling packets with the data packets in a separate $\mathrm{PW}$

- '11' for CESOPSN PWs carrying TDM data and CE application signaling on the same PW 
CAS

MUST be cleared for all types of TDM PWs excluding trunk-specific NxDSO services with CAS. For these services, it encodes the trunk framing like the following:

$$
\begin{aligned}
& \text { '01' - an E1 trunk } \\
& \text { ' } 10 \text { ' - a T1/ESF trunk } \\
& 0 \text { '11' - a T1 SF trunk }
\end{aligned}
$$

RSVD-1 and RSVD-2

Reserved bits, which MUST be set to 0 by the PW endpoint distributing this FEC and MUST be ignored by the receiver.

PT Indicates the value of Payload Type in the RTP header expected by the PW endpoint distributing this FEC. A value of 0 means that the PT value check will not be used for detecting malformed packets.

FREQ Frequency of timestamping clock in units of 8 $\mathrm{kHz}$.

SSRC

Indicates the value of the Synchronization source ID (SSRC ID) in the RTP header expected by the PW endpoint distributing this FEC. A value of 0 means that the SSRC ID value check will not be used for detecting misconnections. Alternatively, Length can be set to 8 in this case.

Notes:

1. This interface parameter MAY be omitted in the following cases:

a) SATOP PWs that do not use RTP header [RFC4553].

b) Basic CESOPSN NxDSO services without CE application signaling [RFC5086].

C) TDMoIP AAL1 mode 0 or 2 PWs that do not use RTP.

d) TDMOIP AAL2 PWs that do not relay CAS signaling and do not use RTP . 
2. This interface parameter MUST be present in the following cases:

a) All TDM PWs that use RTP headers.

b) CESoPSN PWs that carry basic NxDSO services and use CESoPSN signaling packets to carry CE application signaling. This case is discussed in detail in section 4 below.

c) CESOPSN PWs that carry trunk-specific NxDSO services with CAS.

d) TDMOIP AAL1 mode 1 PWs.

e) TDMOIP AAL2 PWs that relay CAS signaling.

3. If RTP header and possibly the Differential timestamping mode are used, the value of the Length field MUST be set to 8 or 12 in order to accommodate the Timestamping Clock Frequency and SSRC fields.

4. Usage or non-usage of the RTP header MUST match for the two directions making up the TDM PW. However, it is possible to use the Differential timestamping mode in just one direction.

4. Extending CESOPSN Basic NxDSO Services with CE Application Signaling

[RFC5086] states that basic NxDSO services can be extended to carry $\mathrm{CE}$ application signaling (e.g., CAS) in special signaling packets carried in a separate $\mathrm{PW}$.

The following rules define the setup of matching pairs of CESoPSN PWs using the PW ID FEC and the extensions defined above:

1. The two PWs MUST:

a) Have the same PW type.

b) Use the same setup method (i.e., either both use the PWId FEC, or both use the Generalized PW FEC).

c) Have the same values of all the Interface Parameters listed in Section 3.1 above with the exception of the code point in the SP field of the TDM Options parameter:

i) For the PW carrying TDM data packets, the SP bits MUST be set to ' $01^{\prime}$.

ii) For the PW carrying the signaling packets, the SP bits MUST set to '10'. 
2. If the PWId FEC has been used:

a) The value of PW ID for the CESOPSN PW carrying TDM data packets MUST be even.

b) The value of PW ID for the CESOPSN PW carrying CE application signaling MUST be the next (odd) value after the (even) PW ID of the CESOPSN PW carrying TDM data packets.

When using the Generalized PW FEC for the setup of the two PWs, no specific rules for matching the two FECs are defined.

Implementation-specific mechanisms MAY be employed to verify the proper matching of the TDM data PW with its associated CE signaling $\mathrm{PW}$.

If one of the two associated PWs has been established and the other failed to be established, or for any reason fails after having been established, the established PW MUST be torn down.

5. LDP Status Codes

In addition to the status codes defined in Sections 5.1 and 7.2 of [RFC4447], the following status codes defined in [RFC4446] MUST be used to indicate the reason of failure to establish a TDM PW:

1. Incompatible bit-rate:

a) In the case of a mismatch of $\mathrm{T} 1$ encapsulation modes (basic vs. octet-aligned).

b) In the case of a mismatch in the number of timeslots for NxDS0 basic services or trunk-specific NxDSO services with CAS.

2. CEP/TDM misconfiguration:

a) In the case of a mismatch in the desired usage of RTP header.

b) In the case of a mismatch of the desired Timestamping Clock Frequency.

c) In the case of a mismatch of expected signaling packets behavior for basic CESOPSN NxDSO services extended to carry CE application signaling in separate signaling packets.

d) In the case of trunk-specific NxDSO services with CAS if the framing types of the trunks are different. 
e) In the case of TDMoIP AAL1 PWs with different AAL1 modes specified by the endpoints.

3. The generic misconfiguration error MAY be used to indicate any setup failure not covered above.

In cases 2a, 2b, 2c, and $2 \mathrm{e}$ above, the user MAY reconfigure the endpoints and attempt to set up the PW once again.

In the case of $2 d$, the failure is fatal.

Note that setting of the Control bit (see Section 2 above) to zero MUST result in an LDP status of "Illegal C-Bit".

6. Using the PW Status TLV

The TDM PW control word carries status indications for both attachment circuits ( $L$ and $M$ fields) and the PSN (R field) indication (see [RFC4553], [RFC5086], and [RFC5087]). Similar functionality is available via use of the PW Status TLV (see Section 5.4.2 of [RFC444]). If the latter mechanism is employed, the signaling PE sends its peer a PW Status TLV for this PW, setting the appropriate bits (see Section 3.5 of [RFC4446]):

- Pseudowire Not Forwarding

- Local Attachment Circuit (ingress) Receive Fault

- Local Attachment Circuit (egress) Transmit Fault

- Local PSN-facing PW (ingress) Receive Fault

o Local PSN-facing PW (egress) Transmit Fault

As long as the TDM PW interworking function is operational, usage of the Status TLV is NOT RECOMMENDED in order to avoid contention between status indications reported by the data and control plane. However, if the TDM PW interworking function (IWF) itself fails while the PWE3 control plane remains operational, a status TLV with all of the above bits set SHOULD be sent.

\section{IANA Considerations}

Most of the IANA assignments required by this document are already listed in [RFC4446]. Additional assignments have been made for four Interface Parameter Sub-TLV types (see Section 3.1):

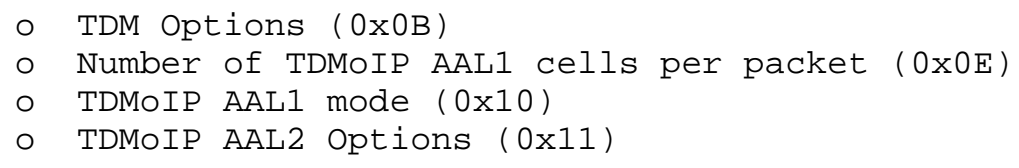




\section{Security Considerations}

This document does not have any additional impact on the security of PWs above that of basic LDP-based setup of PWs specified in [RFC4 4 4 ].

9. Acknowledgements

Sharon Galtzur has reviewed one of the previous versions of this document. Y. (J.) Stein would like to thank Barak Schlosser for helpful discussions.

10. References

10.1. Normative References

[RFC2119] Bradner, S., "Key words for use in RFCs to Indicate Requirement Levels", BCP 14, RFC 2119, March 1997.

[RFC5036] Andersson, L., Ed., Minei, I., Ed., and B. Thomas, Ed., "LDP Specification", RFC 5036, October 2007.

[RFC4447] Martini, L., Ed., Rosen, E., El-Aawar, N., Smith, T., and G. Heron, "Pseudowire Setup and Maintenance Using the Label Distribution Protocol (LDP)", RFC 4447, April 2006.

[RFC4446] Martini, L., "IANA Allocations for Pseudowire Edge to Edge Emulation (PWE3)", BCP 116, RFC 4446, April 2006.

[RFC4623] Malis, A. and M. Townsley, "Pseudowire Emulation Edge-toEdge (PWE3) Fragmentation and Reassembly", RFC 4623, August 2006 .

[RFC4553] Vainshtein, A., Ed., and YJ. Stein, Ed., "StructureAgnostic Time Division Multiplexing (TDM) over Packet (SATOP)", RFC 4553, June 2006.

\subsection{Informative References}

[RFC5086] Vainshtein, A., Ed., Sasson, I., Metz, E., Frost, T., and P. Pate, "Structure-Aware Time Division Multiplexed (TDM) Circuit Emulation Service over Packet Switched Network (CESOPSN)", RFC 5086, December 2007.

[RFC5087] Y(J). Stein, Shashoua, R., Insler, R., and M. Anavi, "Time Division Multiplexing over IP (TDMoIP)", RFC 5087, December 2007 . 
[Q.2630.1] ITU-T Recommendation Q.2630.1, December 1999, AAL type 2 signaling protocol - Capability set 1

[RFC4805] Nicklass, O., Ed., "Definitions of Managed Objects for the DS1, J1, E1, DS2, and E2 Interface Types", RFC 4805, March 2007 .

[RFC4842] Malis, A., Pate, P., Cohen, R., Ed., and D. Zelig, "Synchronous Optical Network/Synchronous Digital Hierarchy (SONET/SDH) Circuit Emulation over Packet (CEP)", RFC 4842, April 2007.

Authors' Addresses

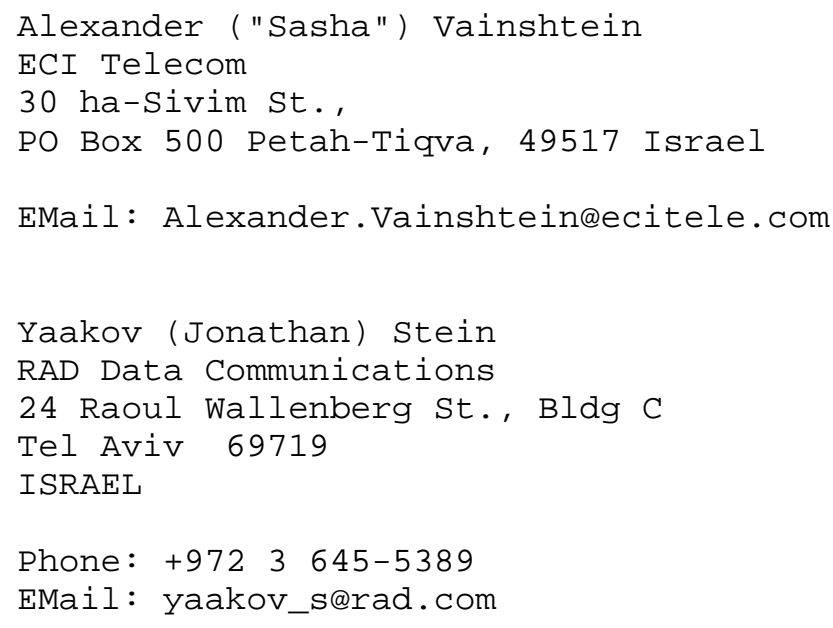


Full Copyright statement

Copyright (C) The IETF Trust (2008).

This document is subject to the rights, licenses and restrictions contained in BCP 78, and except as set forth therein, the authors retain all their rights.

This document and the information contained herein are provided on an "AS IS" basis and THE CONTRIBUTOR, THE ORGANIZATION HE/SHE REPRESENTS OR IS SPONSORED BY (IF ANY), THE INTERNET SOCIETY, THE IETF TRUST AND THE INTERNET ENGINEERING TASK FORCE DISCLAIM ALL WARRANTIES, EXPRESS OR IMPLIED, INCLUDING BUT NOT LIMITED TO ANY WARRANTY THAT THE USE OF THE INFORMATION HEREIN WILL NOT INFRINGE ANY RIGHTS OR ANY IMPLIED WARRANTIES OF MERCHANTABILITY OR FITNESS FOR A PARTICULAR PURPOSE.

Intellectual Property

The IETF takes no position regarding the validity or scope of any Intellectual Property Rights or other rights that might be claimed to pertain to the implementation or use of the technology described in this document or the extent to which any license under such rights might or might not be available; nor does it represent that it has made any independent effort to identify any such rights. Information on the procedures with respect to rights in RFC documents can be found in BCP 78 and BCP 79 .

Copies of IPR disclosures made to the IETF Secretariat and any assurances of licenses to be made available, or the result of an attempt made to obtain a general license or permission for the use of such proprietary rights by implementers or users of this specification can be obtained from the IETF on-line IPR repository at http://www.ietf.org/ipr.

The IETF invites any interested party to bring to its attention any copyrights, patents or patent applications, or other proprietary rights that may cover technology that may be required to implement this standard. Please address the information to the IETF at ietf-ipreietf.org. 